\title{
What Causes Late Perforation of the Jejuno-Jejunal Anastomosis After Roux-en-Y Gastric Bypass Surgery?
}

\author{
Dino Kröll ${ }^{1}$ • Arnold Kohler ${ }^{2}$ Philipp Christoph Nett ${ }^{1}$
}

Published online: 23 August 2015

(C) Springer Science+Business Media New York 2015

To the Editor:

Laparoscopic Roux-en-Y gastric bypass (RYGB) is a common surgical treatment for morbid obesity that is being used more and more frequently and is still widely regarded today as the gold standard in weight loss surgery. There are, however, a number of short-term and long-term sequelae that may occur after surgery. Complications related to this intervention occurring more than 30 days postoperatively and requiring readmission are defined as late complications. These select intestinal complications include small-bowel obstructions (the most frequently reported late complication), hernias, anastomotic strictures, and marginal or stomal ulceration [1]. Late perforation at the site of the jejunojejunal (JJ) anastomosis appears to be very rare. The incidence of $\mathrm{JJ}$ anastomotic perforation has been reported to be under $1 \%$ in the literature $[2,3]$. To our best knowledge, only ten cases have been reported following bariatric surgery, five of them in Obesity

Dino Kröll

dino.kroell@insel.ch

Arnold Kohler

arnold.kohler@spitalfmi.ch

Philipp Christoph Nett

philipp.nett@insel.ch

1 Department of Visceral Surgery and Medicine,

Bern University Hospital and University of Bern, Switzerland, 3010 Bern, Switzerland

2 Department of Surgery, FMI Hospital Interlaken, Switzerland, 3800 Interlaken, Switzerland

Surgery, and the reason for perforation has not always been apparent [2-7].

We would like to present an additional case of late perforation at the JJ anastomosis, which occurred 11 months after laparoscopic RYGB performed at another hospital in a patient with an initial BMI of $42 \mathrm{~kg} / \mathrm{m}^{2}$. We would appreciate hearing about similar observations made by other clinicians.

A 36-year-old woman with a total excess weight loss (EWL) of $30 \%$ was admitted to the emergency department with severe, cramping abdominal pain and severe diarrhea. Clinical examination revealed a tender abdomen. Laboratory findings were normal except for an elevated leukocyte count of $14,700 / \mu l$. Free air in the abdomen was detected by $\mathrm{CT}$ scan, and the patient was immediately transferred to the operating room. After initial exploratory laparoscopy, an open technique was applied because of extensive peritonitis. A 5-mm perforation was identified at the mesenteric site of the JJ anastomosis. Specimens were obtained for microbiological cultures. The perforation was excised, and primary closure was performed with interrupted absorbable sutures. Intravenous antimicrobial agents and proton pump inhibitors were administered for 14 days. The patient denied taking any ulcerogenic drugs, consuming alcohol, and smoking. Streptococcus gallolyticus, Klebsiella oxytoca, and Aeromonas hydrophila grew in culture. The postoperative course was favorable.

In previous reports, potential causes of perforation have included the following: distal obstruction (increased intraluminal pressure; caused by a bezoar [6] 
in one report), anastomotic weakness, and suture degradation or abscess formation [2, 4]. Certain risk factors, such as ulcerogenic drugs, alcohol consumption, smoking, and the presence of Helicobacter pylori, can contribute to perforation.

In our case, no obvious reason for the perforation was found. The relevance of the microbiological findings is difficult to interpret because they may represent the patient's flora.

However, tissue damage caused by cytotoxic bacteria is possible. Both K. oxytoca and A. hydrophila can produce pore-forming toxins that cause significant tissue damage at the local infection site. Moreover, A. hydrophila may cause severe gastrointestinal disease, such as that observed in our patient. Nevertheless, these microorganisms' potential to cause tissue necrosis does not prove that they caused the perforation in our case.

We kindly ask experts in the field of obesity surgery to share their experiences regarding the following issues. First, is late perforation of the JJ anastomosis indeed an extremely rare occurrence or does it rather represent an underreported complication after RYGB? Second, given that anastomotic ischemia is thought to be the most likely cause of ulceration, does the JJ anastomosis represent a locus minor resistentiae for late intra-abdominal complications? Finally, considering that microbiological sampling after perforation may not be meaningful and is rarely performed, what is the role of microorganisms (changes in the gut microbiota) in causing perforation?
Conflict of Interest The authors declare that they have no competing interests.

Statement of Informed Consent Informed consent was obtained from all individual participants included in the study.

Statement of Human Rights All procedures performed in the study involving human participants were in accordance with the ethical standards of the institutional research committee and with the 1964 Helsinki Declaration and its later amendments or comparable ethical standards.

Financial or Material Support None.

\section{References}

1. Livingston EH. Complications of bariatric surgery. Surg Clin North Am. 2005;85(4):853-68.

2. Goitein D, Papasavas PK, Gagne DJ, et al. Late perforation of the jejuno-jejunal anastomosis after laparoscopic Roux-en-Y gastric bypass. Obes Surg. 2005;15(6):880-2.

3. Kalaiselvan R, Abu Dakka M, Ammori BJ. Late perforation at the jejuno-jejunal anastomosis after laparoscopic gastric bypass for morbid obesity. Surg Obes Relat Dis. 2013;9(6):874-8.

4. Gonzalez-Pezzat I, Soto-Perez-de-Celis E, Pantoja-Millan JP. Late perforation and abscess formation at the site of the jejunojejunal anastomosis following laparoscopic roux-en-Y gastric bypass surgery. Obes Surg. 2009;19(5):661-3.

5. Spanager L, Sigild UH, Neuenschwander AU. Late perforation following laparoscopic gastric bypass. Ugeskr Laeger. 2010;172(36): 2440-1.

6. Sammut SJ, Majid S, Shoab S. Phytobezoar: a rare cause of late upper gastrointestinal perforation following gastric bypass surgery. Ann R Coll Surg Engl. 2012;94(2):e85-7.

7. Anderson PL, Velanovich VV, Kaufman CR, et al. Late perforation of the distal Roux-en-Y anastomosis in a patient with biliopancreatic diversion. Obes Surg. 1992;2(3):269-70. 